\title{
A Comparative Study of Norwegian and Ethiopian Textbooks: The Case of Relations and Functions Using Anthropological Theory of Didactics (ATD)
}

\author{
Solomon A. Tesfamicael", Øyvind A. Lundeby \\ Department of Teacher Education (ILU), Norwegian University of Science and Technology (NTNU), Norway
}

Copyright@2019 by authors, all rights reserved. Authors agree that this article remains permanently open access under the terms of the Creative Commons Attribution License 4.0 International License

\begin{abstract}
This paper provides a comparative study of mathematics textbooks as the primary construct via the Anthropological Theory of Didactics (ATD), which was founded by Yves Chevallard. The aim is to suggest principles that can be incorporated to improve the designing of mathematics textbooks. Definitions, examples, and tasks dealing with the teaching and learning of relations and functions, as sub-construct, from several mathematics textbooks in Norway and one textbook from Ethiopia was collected and analyzed using didactical tools such as types of representations, cognitive demand levels, and response types of mathematical tasks. In the findings, similarity and differences of textbooks, both within homogeneous and heterogeneous cultures, were adequately identified and explained in a way that the results contribute to the improvement of the intended curriculum. In doing so, it shows that ATD provides an excellent tool for textbook design research in the future.
\end{abstract}

Keywords Comparative studies, Textbooks, Relations and Functions, Anthropological Theory of Didactics

\section{Introduction}

Comparing is one of the primary intellectual activities of human beings. We continuously make comparisons to understand where we stand, both in relation to others as well as to our own past experiences [1]. Comparative studies include studies that document, analyze, contrast, or juxtapose similarities and differences across all aspects and levels of mathematics education [2] in a broader sense. Artigue and Winsløw [3] argue that comparative studies are useful in investigating similarities and differences of one construct in two different contexts. In this study, textbooks (as the primary construct) from Norway and
Ethiopia (as two different contexts) are considered.

Comparative studies of education help schoolteachers, educators, policymakers, the broad public, and the research community to improve the quality of education by facilitating the dissemination of different useful frameworks, principles, and models across nations [4]. Cai et al. [1] have mentioned four objectives of comparative studies: to identify what is happening in different countries that might help improve education systems and outcomes; to describe similarities and differences in educational phenomena between systems of education and interpreting why these exist; to estimate the relative effects of variables that are thought to be determinants of educational outcomes; to identify general principles concerning educational effects. The rationale to do a binary comparative study in mathematics education in Norway and Ethiopia lies within these objectives. The goal is not only to reflect on their practices in light of international wisdom [5], but also to lay down grounds for further intervention studies. For example, the Norwegian NORHED project, started in 2017 (https://www.norad.no/en/front/funding/norhed/news/) [6]; also to go beyond projects and contribute to the improvement of textbooks for effective teaching and learning of mathematics in school.

\subsection{Main Construct: Textbooks}

Textbooks play a crucial role in the teaching and learning of mathematics. They can serve as tools, artifacts, or instruments that facilitate the daily work of teachers and also provide learning opportunities for students. They are the mediators between intention and implementation [7] (see Figure 1). They contribute to the field of mathematics by preserving and transmitting skills and knowledge [8]. In general, mathematics is a subject that has long been associated with textbooks and curriculum materials [9]. 


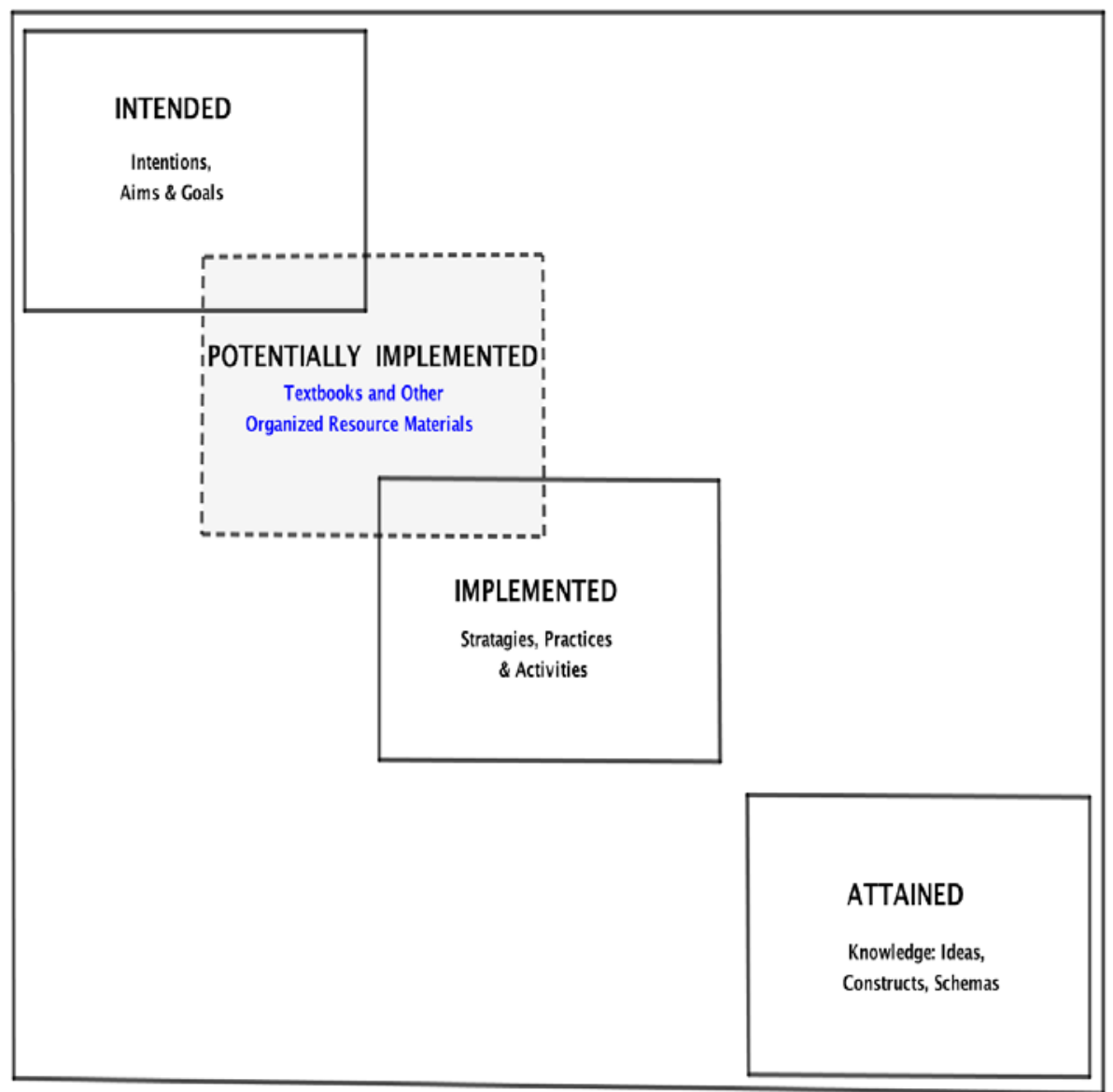

Figure 1. Tripartite Curriculum Model The International Association for the Evaluation of Educational Achievement (IEA) [9]

Regardless of how teachers or students use their textbooks, how mathematical concepts are communicated, or how the design of mathematics textbooks are done in different contexts, textbooks can be used as a construct for comparison [10]. By reporting how textbooks are used by teachers in England, France, and Germany, Haggarty and Pepin [11] argue that textbooks are among the several constructs that influence the teaching and learning cultures of the mathematics classroom. In fact, they are the main resources of many mathematics classrooms in many countries [9,12].

In this study, the mathematics textbook comparison in Ethiopia and Norway is conducted by focusing on the teaching and learning of relations and functions. Several mathematics textbooks from Norway that deal with these mathematical concepts are included due to their availability. Hence, the study not only provides a comparison of different cultural contexts but also offers insights into how textbooks are prepared in a homogeneous culture, how they are written at different times and by different people and publishers. The study has, therefore, a dual purpose.

\subsection{Sub-construct: Relations and Functions}

For two main reasons, emphasis is given to the teaching and learning of relations and functions as presented in the textbooks: first, for the sake of limiting the scope of the study and, second, due to students' difficulties in learning these topics [13-15]. As teacher educators working with students who are preparing to work in primary and middle schools in Norway, we have also observed that many student teachers struggle to grasp these concepts and teach them. Understanding such mathematical concepts can be boosted by including different essential features and findings from recent research in mathematics education in the preparation of textbooks. This is one of the purposes of this study - to improve textbook development to enable better learning opportunities for students.

Viirman's [16] research on the concept of function within mathematics education provides its historical development with some didactical reflections. Viirman argued that research on this concept has tended to reveal difficulties related to its learning. Different approaches, both from the cognitive and sociocultural perspective, have been applied to achieve a better understanding. One alternative is to use a set-theoretical definition, which is problematic for learners, and another is to use multiple representations that minimize the abstraction level of the teaching and learning of the concept [16,17]. Furthermore, Stein and Smith [18] emphasize the importance of choosing exercises or activities that challenge students to 
think, justify, explain, and find meaning-exercises that stimulate them to make connections [19]. Textbooks should provide such opportunity to the learners. These insights are used to analyze the textbooks chosen for this study.

The researchers-one originally from Ethiopia with teaching experience of these topics at a lower middle school and now living in Norway, and another Norwegian with many years' experience in teaching the topics for several years-had the opportunity to grasp these constructs (textbooks and mathematical topics of relations and functions). Besides, we have been working on the teaching-learning of the topic of function for more than five years at the teacher education programs in Norway. Given this background, the main research question asked is this: What are the similarities and differences of Norwegian and Ethiopian textbooks in dealing with the topic of relations and functions in light of the analytical frameworks designed in the study? More specifically, how are these concepts represented in the textbooks? Which level of cognitive demand dominates the mathematical tasks? Are the exercises more open- or closed-ended? To answer this question, definitions, examples, and exercises used in the textbooks are given due attention, since the purpose is not to compare the physical features of the textbooks, but instead on the learning opportunities provided by the textbooks. Next, the analytical framework used for collecting evidence and comparing the constructs is presented.

\section{Analytical Framework}

This binary comparison didactic phenomenon is viewed from the perspective of the ATD via the simple model proposed by Chevallard [20]. The use of ATD in this work has multimodal purposes. First, since it provides a framework that enables to be explicit about what is compared and what is not via the levels of didactic co-determination, ATD helps to situate the comparative study at hand [3, 21]. Second, it provides a tool to analyze the data in the study, since ATD through praxeology can be a model to analyze both mathematical and didactical knowledge. Third, it can also be used as a designing tool for preparing textbooks [22, 23]. Finally, it assists to state the findings of the study together with its limitations. Therefore, a short presentation of ATD is overdue, and for better understanding, the reader is referred to [21] and other references mentioned here.

Chevallard [24] introduced ATD, a new mathematics didactic theory that can be used to investigate human mathematical activities. It hypothesizes that any activity related to the production, diffusion, or acquisition of knowledge should be interpreted as an ordinary human activity. Chevallard modeled the activities via the practice and knowledge block (see Figure 2). The practice block, as the minimum human activity, consists of a task (T) and technique $(\tau)$ used to achieve or solve the task. Hence, the practice block is a tuple $(T, \tau)$. Note that task is defined here in a very general sense. As Chevallard puts it, "irrespective of its volume or pettiness: to open this door and to smile to this neighbor are tasks; to scratch this person's back, to write this sonnet, to save this polar bear, to prove this theorem, and to play this guitar chord are tasks as well,” whereas, a technique $(\tau)$ is a way to execute or to accomplish the task $\mathrm{T}$ [25].

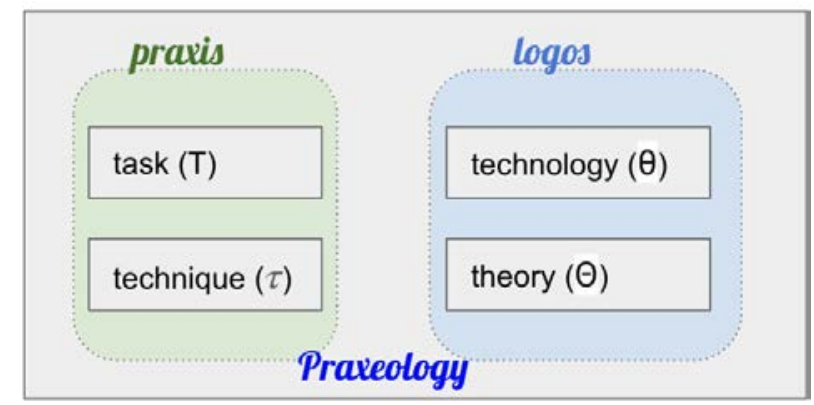

Figure 2. The practical (praxis) block and the knowledge (logos) block of the praxeology. It is called punctual organization under the ATD.

The knowledge block is made of a technology $(\theta)$ to justify the technique $(\tau)$ and a super discourse on the technology, or a wider discourse explaining the technology $\theta$, called theory $\Theta$. Chevallard et al. explain technology as follows:

This word is used in ATD with its etymological value: as the suffix -logy indicates, a technology is a "discourse" on a given technique $\tau$. This discourse is supposed, at least in the best-case scenario, both to justify the technique $\tau$ as a valid way of performing tasks $\mathrm{T}$ and to throw light on the logic and workings of that technique $\tau$, making it at least partially intelligible to the user [25].

That is, the knowledge block is a tuple $(\theta, \Theta)$. Altogether, the theory of praxeology (fusion of "praxis" and "logos") is of a quadruple (T, $\tau, \theta, \Theta)$ and, thus, the praxeology block models an amalgam of human practice and knowledge $[3,21,23,26,27]$.

Let us give a particular example of such modeling within the topic of the teaching of relations and functions. Consider that students are given the task (T) of finding the null points of a quadratic function, $f(x)=a x^{2}+b x+c$. The algorithm used to find the zeros of the function is called technique $(\tau)$. The explanations, like why putting the function value to zero and the like can be considered as technology $(\theta)$, while the whole discourse about functions can be viewed as theory $(\Theta)$. Another example in line to this work, let the task $\mathrm{T}$ be to represent the concept of function. Five different ways, or called here as techniques $\tau$, are used by Lesh et al. [28]: visual, verbal, symbolic, physical, and contextual to achieve the goal. The explanations and discussion around each technique and, in general, around the use of mathematical representation can 
be structured as "logos" block in Figure 2 above. In this paper, an expanded version of this is discussed below.

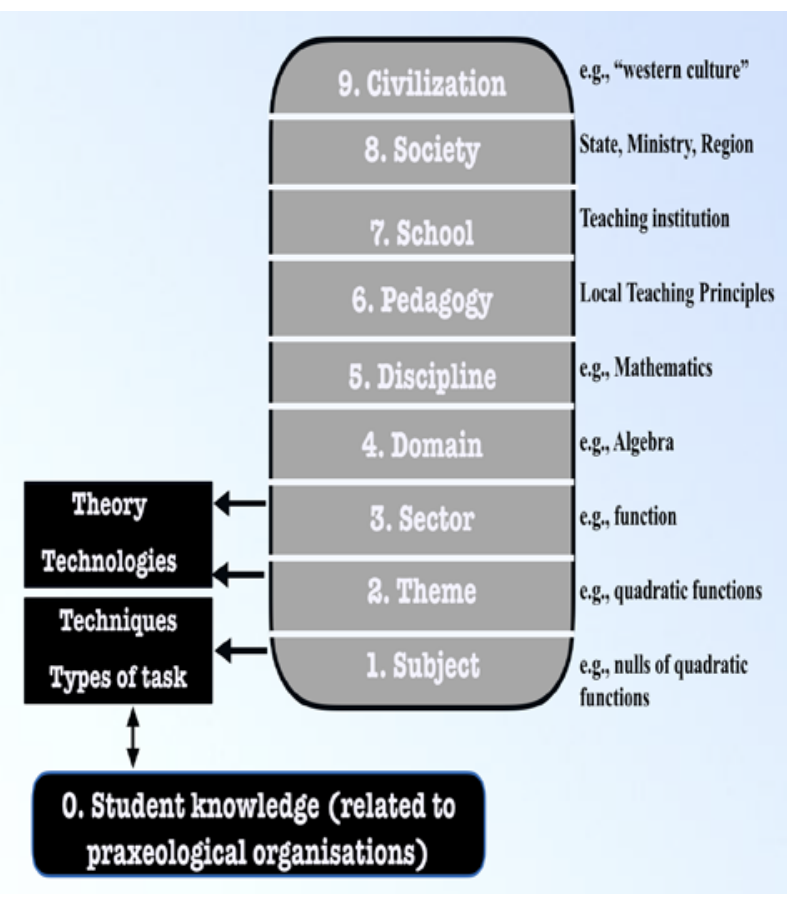

Figure 3. Levels of didactic co-determination as proposed by Chevallard [20] as adopted for this work.

Levels of didactic co-determination (see Figure 3), proposed by [20], is a simple, elegant model that gives a zoomed insight into the Tripartite Curriculum Model of IEA in Figure 1. The model shows the interactions among each neighboring level as well as the praxeological model of "what happens in the classroom" [21] or, in our case, how textbooks diffuse knowledge. It has both supra-disciplinary (the pedagogy, the school institution, the society, and the civilization) and sub-disciplinary (domain, sector, theme, and subject) levels of didactic co-determination (with the co-indicating the solidaric relation between didactic and mathematic organization). Artigue et al. [3] showed a detailed analysis regarding how this model can be used in comparative studies, from large-scale surveys like The Programme for International Student Assessment (PISA) and Trends in International Mathematics and Science Study (TIMSS) to small-scale binary comparisons in doctoral projects. They provide a synthetic analysis of a selected body of international comparative research on mathematics education with the particular aim of understanding how the different studies differ and relate [3]. We are inspired by their work to apply it in this binary comparative study.

Families of praxeologies unified by a technology $\theta$ are called a local organization (LO), and those bound together by a theory $\Theta$ are called a family of a regional organization (RO) [3]. For example, the discourse about polynomial functions is LO, and the theory of functions can be RO. If the task involved is mathematical, such organizations are called mathematical organization (MO). Further, such praxeological organizations are coupled with didactic phenomena, and they form didactic organizations (DO) (punctual, local and regional, in the same way as MOs) (Winsløw, 2011). As Chevallard puts it, these two, DO and MO, co-determine with each other with respect to the "sub-disciplinary" levels (see Figure 3). Moreover, the didactical and mathematical praxeologies and their interaction cannot be fully grasped without situating them within the broader context of a whole hierarchy of institutional levels (Figure 3) as presented by Chevallared (Artigue, 2010; Winsløw 2011).

Since textbooks help diffuse/transfer of the intended curriculum to the learners, this activity is modeled via the praxeology tool of ATD. In line with the praxeology block in Figure 2, four tasks $T_{1}, T_{2}, T_{3}$, and $T_{4}$ are selected among different principles that help design a mathematics textbook. $T_{1}$ is about the type of representation used for definitions, examples, and exercises of a mathematical concept such as relations and functions. $T_{2}$ is all about the cognitive demand level of the exercises in the textbooks. $T_{3}$ deals if the exercises/problems/tasks are open- or closed-ended response types, while $T_{4}$ deals with the approach taken to relations and functions in the textbooks. In turn, all these tasks have different techniques or ways to achieve the tasks defined. In other words, four didactical LOs, around a respective didactical technology, are selected as part of the analytical framework. These are representations (technology $\theta_{1}$ ), cognitive demand level (technology $\theta_{2}$ ), type of responses (technology $\theta_{3}$ ), and discourse (technology $\theta_{4}$ ), which is about the structure of the content of a textbook [7].

\subsection{Web of Representations-Local Organization 1}

Effective teaching of mathematics engages students in making connections among mathematical representations [29]. Semiotic representations in textbooks, if they are well-described and directed, can help learners effectively construct meanings of a mathematical concept [30]. Textbooks should provide an opportunity for learners to utilize different representations of mathematical concepts. Understanding the concept of function from cognitive perspectives implies an ability to make connections between the different representations of the concept [31]. Using and connecting different representations are important ways to nurture students' understanding of mathematical concepts. Lesh et al. [32] indicated that students who have difficulty in translating a concept from one representation to another have difficulty in applying the concept in problem solving and computations. The principle of representation is crucial, and that is why [33] include it among the five most important process standards of school mathematics [34]. This discourse is our first didactical local organizations (LO1) used around the technology representations $\left(\theta_{1}\right)$ used to analyze the textbooks considered in the research.

Different representations peculiar for functions and the 
translation between was given by Janvier et al. [35] and Lesh et al. [32]. An expanded form, consisting of seven different representations called web of representations, are provided in the book called Elementary and Middle school mathematics [36]. In Figure 4, the summary of the representations and their interconnectedness, as well as the seven techniques $(\tau)$, are shown. These are giving a context $\tau_{1}$, explain meaning in words $\tau_{2}$, illustrate with physical tools $\tau_{3}$, write using symbols $\tau_{4}$, draw a diagram $\tau_{5}$, display data in a table $\tau_{6}$, and create a graph $\tau_{7}$. These techniques used for definitions, examples, and tasks in the textbooks are counted, analyzed, categorized, and compared.

Effective teaching of mathematics engages students in solving and discussing tasks that promote mathematical reasoning and problem solving and allow multiple entry points and varied solution strategies [29]. Further, the nature of the mathematical tasks to which students are exposed determines what students learn [37].

Tasks can be routine, simple, complex, or challenging. This is another local organization around a didactical technology called cognitive demand levels ( technology $\theta_{2}$ ). Smith and Stein [18] and other authors have developed four different levels of tasks based on cognitive demand. They are rebranded as techniques to make tasks under the didactic technology. In this study, these techniques of preparing tasks are branded as follows: Lower-level demands I is memorization $\tau_{8}$, lower-level demands II is procedures without connections $\tau_{9}$, higher-level demands I is procedures with connections $\tau_{10}$, and higher-level demands II is doing mathematics $\tau_{11}$, where each are described as in Figure 5, adapted from [29].

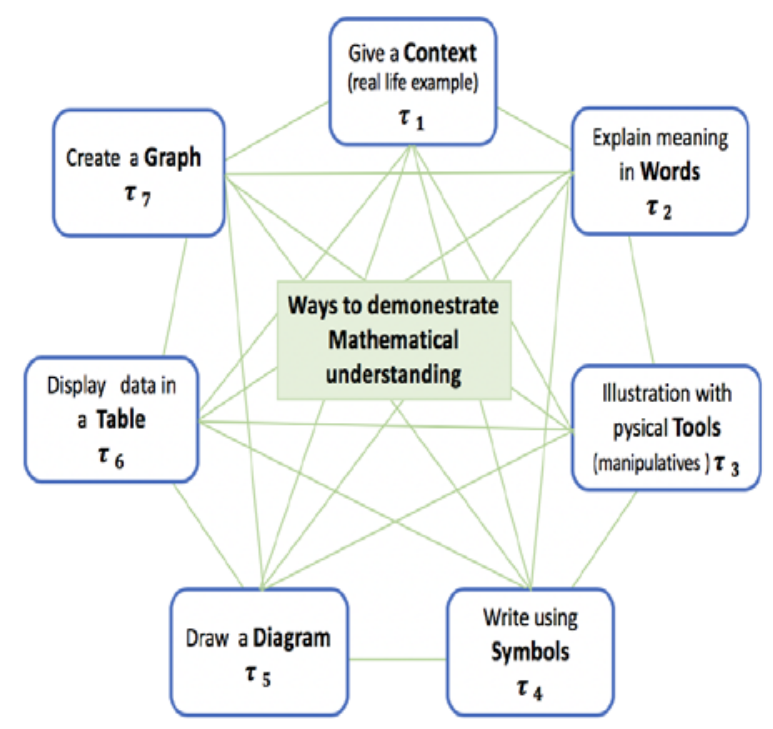

Figure 4. Web of representations together with 7-technique $\tau_{1}-\tau_{7}$, adopted from Van et al. (2015, p 45). Using and connecting the different mathematical representations demonstrates mathematical understanding (NCTM, 2014). 2.2. Cognitive Demand Levels-Local Organization 2

\begin{tabular}{|c|c|}
\hline $\begin{array}{l}\text { Lower-Level demand I } \\
\left.\text { (Memorization }-\tau_{\mathrm{B}}\right)\end{array}$ & $\begin{array}{l}\quad \text { Lower-Level demand II } \\
\quad \text { (Procedures without connections - } \tau_{9} \text { ) } \\
\text { - are algorithmic } \\
\text { - require limited cognitive demand } \\
\text { the po connection to concepts or meaning that underline } \\
\text { - Focus on producing correct answers instead of understanding } \\
\text { - Require no explanations or explanations that focus solely on } \\
\text { describing the procedure }\end{array}$ \\
\hline $\begin{array}{l}\text { Higher-Level demand I } \\
\left.\quad \text { (Procedures with connections }-\tau_{10}\right) \\
\text { - use procedure for deeper understanding of concepts } \\
\text { - Broad procedures connected to ideas instead narrow } \\
\text { algorithms } \\
\text { - Usually represented in different ways } \\
\text { - Require some degree of cognitive effort. Although } \\
\text { general procedures may be followed, they cannot be } \\
\text { followed mindlessly. }\end{array}$ & \begin{tabular}{l}
\multicolumn{1}{c}{$\begin{array}{c}\text { Higher-Level demand II } \\
\text { (Doing Mathematics }-\tau_{\text {I1) }}\end{array}$} \\
- require complex non-algorithmic thinking \\
- require students to explore and understand the mathematics \\
- demand self-monitoring of one's cognitive process \\
Require considerable cognitive effort and may involve some \\
level of anxiety \\
Require students to analyze the task and actively examine \\
task constraints
\end{tabular} \\
\hline
\end{tabular}

Figure 5. The four cognitive demand levels as techniques $\tau_{8}-\tau_{11}$ adapted from the National Council of Teachers of Mathematics [29].

\section{Closed-ended tasks $-\tau_{12}$}

- have one correct answer

- can be completed quickly

- Assess one specific piece of knowledge, or a specific skill or procedure

- Provide limited information about student thinking

- Provide limited opportunity for students to demonstrate higher levels of understanding

\section{Open-ended tasks - $\tau_{12}$}

- have a range of appropriate responses

- take longer to complete

- assess a range of knowledge and skills

- provide information about problem-solving strategies and thinking

- provide an opportunity for students to demonstrate higher levels of understanding. 


\subsection{Response Types-Local Organization 3}

Another inherent property of mathematical tasks is how tasks are designed so that they offer multiple opportunities for the teaching and learning of particular mathematical concepts [38]. Some tasks can be open- or closed-ended response types (see Figure 6). Open-ended tasks can promote communication [18] and provide an opportunity for teachers to probe and enhance students' mathematical thinking [29]. This is the third local organization around a didactical technology called response type (technology $\theta_{3}$ ), and we call the open- or closed-ended tasks as techniques $\tau_{12}$ and $\tau_{13}$, respectively.

\subsection{The Approach in which the Content of a Textbook Can Unfold-Local Organization 4}

Valverde et al. argue that textbooks differ in the patterns of presentation of their various elements and in the way in which various elements are integrated with each other. The following is quoted directly from their work:

Textbook form and structure advance a distinct pedagogical model. That is, they embody a plan for the particular succession of educational opportunities considered optimal for enacting curricular intentions. An understanding of the form and structure of textbooks permits uncovering the model of instruction that textbooks embody and provides a first examination of how textbooks promote distinctive configurations of educational opportunities in the classroom [7].

We agree that the choice of structure and approach to a topic reflects the kind of discourse at Chevallard's different levels of didactic co-determination (Figure 3). Focusing on the present mathematical topic, the topics of relations and functions, it is clear that the former is broader in scope than the latter, i.e., functions are special types of relations between two variables, magnitudes, or values. This means that the topic of relation can be presented first before arriving at the concept of functions. Function can be an inconvenient concept for learners; it can even be difficult to give a single definition of functions at times [15]. This is our position; the more one understands the relation concept, the better one can grasp the concept of function. Therefore, a new technology (justification or explanation) under the ATD framework, called the approach to relations and functions and denoted as $\theta_{4}$, is introduced in our work. That is, the approach to the topics is the fourth local organization considered in our analysis. In addition, we also identify the prior knowledge for the teaching and learning of the topics. Next, we provide how data is collected.

\section{Method}

In order to choose which textbooks to analyze, a simple survey was performed. We asked a question to teachers about the mathematics textbooks they use for the topic relations and functions in Norway. Our question is: which textbook do you use to teach relations and functions? It was posted/shared in a Facebook group of around 10,000 teachers. A total of 119 teachers responded, and the results are given in Table 1 below.

Table 1. Textbooks used by teachers for relations and functions in Norway (actually they use the mentioned textbook for other topics too)

\begin{tabular}{|c|c|c|}
\hline Book & How many teachers use it & Percent \\
\hline Faktor & 30 & $25,2 \%$ \\
\hline Grunntall & 27 & $22.70 \%$ \\
\hline Maximum & 16 & $13.40 \%$ \\
\hline Tetra & 15 & $12.60 \%$ \\
\hline Nummer & 9 & $7.60 \%$ \\
\hline Mega & 7 & $5.90 \%$ \\
\hline Sirkel & 5 & $4.20 \%$ \\
\hline Others & 10 & $8,4 \%$ \\
\hline Totally & 119 & $100 \%$ \\
\hline
\end{tabular}

Since Faktur is used by more teachers and it is one of the oldest from the list, we chose it for our document analysis work. Unlike the others, Sirkel presents the topics relations and functions separately: relations in $8^{\text {th }}$ grade and functions in $10^{\text {th }}$ grade; for a reason explained later, it is also included in our data analysis. In addition, Maximum is selected in the study since it was written recently (around 2014), and it might be influenced by the recent mathematics education reform called "the New Math," described in [39]. There is only one textbook from Ethiopia for the lower secondary level that covers relations and functions provided by the government. That is, in $9^{\text {th }}$ grade mathematics, the concepts of relations and functions are presented. This book, therefore, is included in our comparative study.

In the selected textbooks, three main categories of content in the textbooks are identified: definitions, examples, and exercises (mathematical tasks). By taking the whole chapter dealing with given mathematical concepts in all the selected textbooks, we characterized and scrutinized it via the analytical tools presented above in the ATD setting. First, the definitions, examples, and exercises are grouped according to the kind of techniques used to represent them. Delineating these as one of the semiotic representations can be difficult at times, but a good deal of effort is induced to be consistent. Consider Figure 7, where the extract is taken from the $9^{\text {th }}$ grade mathematics book from Ethiopia. Both the definition and the example are given using words $\tau_{2}$ and symbols $\tau_{4}$, while in the Sirkel $10^{\text {th }}$ grade textbook (see in Figure 8), three of the representations are applied: word $\tau_{2}$, table $\tau_{6}$, and context $\tau_{1}$ (time versus distance of a scooter). 


\section{Definition 4.1}

Let $A$ and $B$ be non-empty sets. $A$ relation $R$ from $A$ to $B$ is any subset of $A \times B$. In other words, $R$ is a relation from $A$ to $B$ if and only if $R \subseteq(A \times B)$.

Example 1 Let $\mathrm{A}=\{1,2,3,4\}$ and $\mathrm{B}=\{1,3,5\}$

i $\quad R_{1}=\{(1,3),(1,5),(2,3),(2,5),(3,5),(4,5)\}$ is a relation from $A$ to $B$ because $R_{1} \subseteq(A \times B)$. Is $R_{1}$ a relation from $B$ to $A$ ? Justify.

Figure 7. Definition of relation, and one example as it stands in $9^{\text {th }}$ grade mathematics textbook.

Second, the tasks are inspected according to the cognitive demand level required by the students to solve them. Deciding the cognitive demand level of a given task is not an easy task. Deciding the cognitive demand level of a task for students by the researchers may not be similar to the one decided by each learner. However, the criteria from NCTM (2014) listed in Figure 4 are applied strictly to our ability.

Third, the number of open and closed tasks are counted in the selected textbooks. The benchmark for categorizing tasks according to open- or closed-ended is given in Figure 5. Finally, a fourth analytical tool from a textbook design aspect is used to compare via ATD - that is, by studying the approach in which the textbooks entertain the concepts of relations and functions. Next, the whole data analysis is presented.

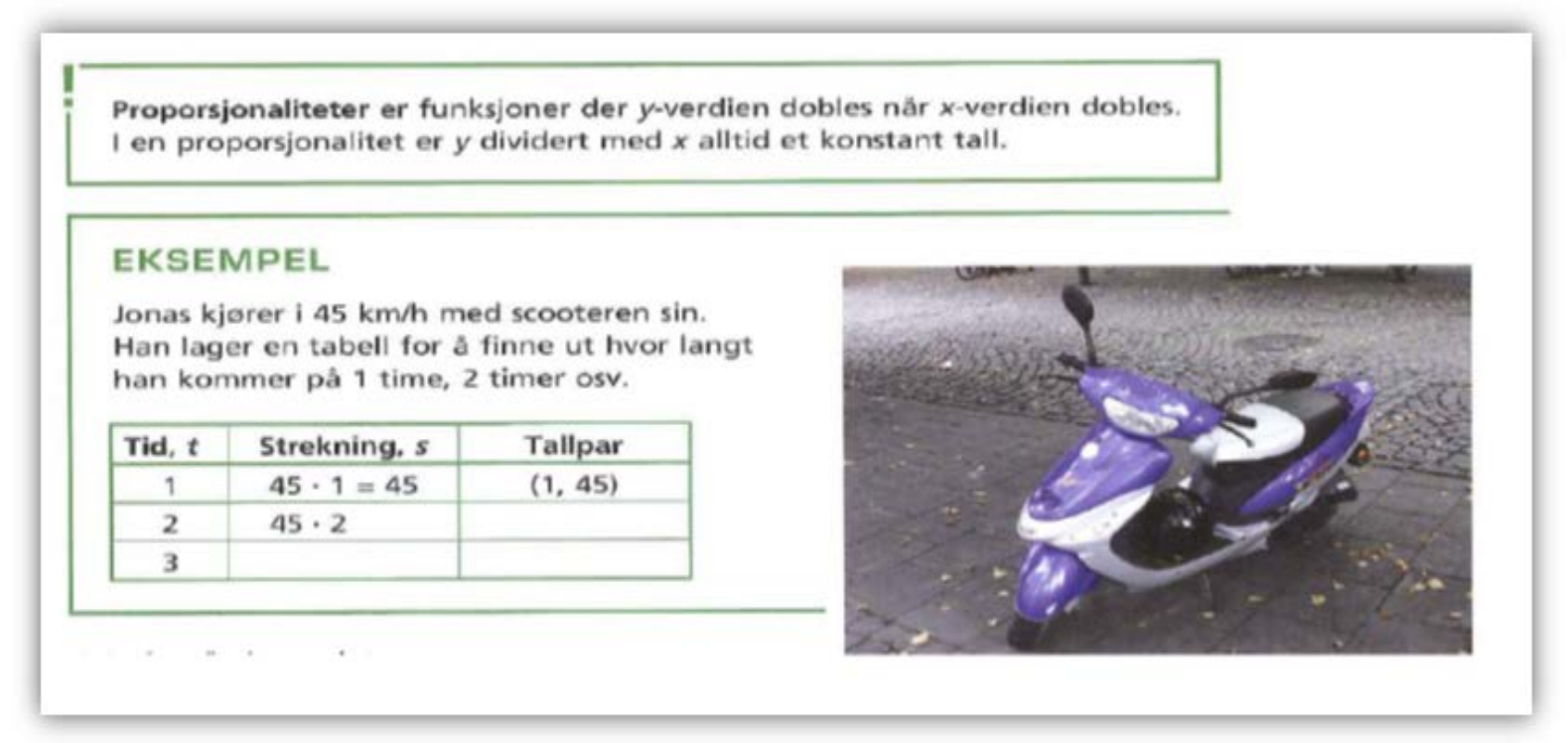

Figure 8. The introduction of function in the textbook called Sirkel (10th grade) using the concept of proportionality and a real-life context example of the speed of a scooter versus time.

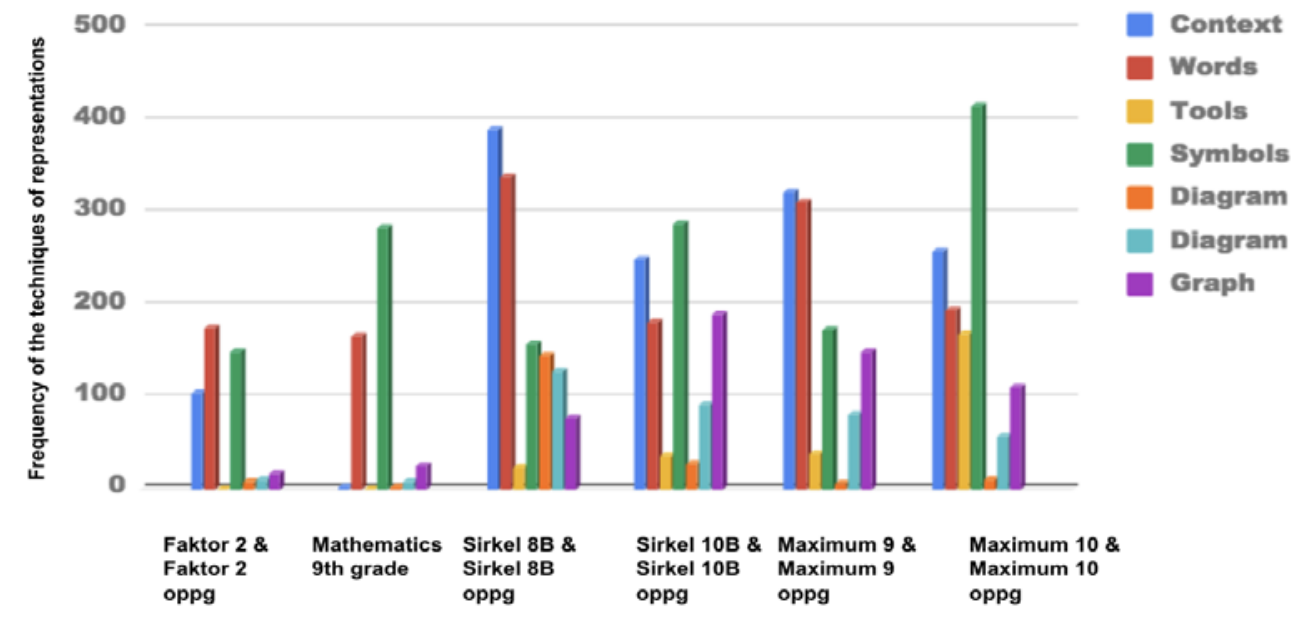

Figure 9. Diagram showing the frequency of the different techniques $\tau$ of representations (i.e., number of words, physical tools, symbols, diagrams, tables, and graphs). 


\section{Comparative Data Analysis and Discussions}

\subsection{Comparison Using the Technology Representation- Local Organization 1}

The way textbooks communicate relations and functions is the first tasks $T_{1}$. The web of representation described above is the techniques, which include the seven different techniques. These families of praxeology are unified by the technology $\left(\theta_{1}\right)$ called representations. This is the first local organization (LO1) used for analyzing definitions, examples, and exercises in the textbooks. Now, how many times do these different techniques $\tau$ are used within each textbook ? Figure 9 provides the summary of the number of times these techniques are used in the chapters of the textbooks that deal with the mathematical concepts under consideration. In Faktor, the techniques context $\tau_{1}$, words $\tau_{2}$ and symbols $\tau_{4}$ dominate, while the frequency of the other four techniques are very low. In the Ethiopian $9^{\text {th }}$ grade mathematics textbook, words $\tau_{2}$ and symbols $\tau_{4}$ are almost the major techniques used with addition of few graphs $\tau_{7}$. The Norwegian textbooks Sirkel $\left(8^{\text {th }}\right.$ and $10^{\text {th }}$ grade) and Maximum $\left(9^{\text {th }}\right.$ and $10^{\text {th }}$ grade) follow a similar pattern in applying these techniques approximately. The techniques context $\tau_{1}$ and words $\tau_{2}$ frequency is higher in both Sirkel 8B and Maximum 9, while in Sirkel 10B and Maximum 10, the use of the technique symbols $\tau_{4}$ is more pronounced. Moreover, the chapters that deal with the concept of relations and functions are counted and analyzed with the chosen framework.

A common feature of all the textbooks is the dominance of the application of the techniques words $\tau_{2}$ and symbols $\tau_{4}$, which contributes to the abstractness of the discipline at hand. But the textbooks differ when it comes to the contextual representation (using context $\tau_{4}$ ). The Ethiopian $9^{\text {th }}$ grade mathematics textbook is almost void of using a real-life example (context $\tau_{1}$ ). Literally, one can say that, context $\tau_{1}$ is not used in the $9^{\text {th }}$ grade mathematics book of Ethiopia in most part of the chapter that deals with the topic of function. Only few such tasks were given using the context of mobile use and expense. In contrast, the Norwegian textbooks entertain the use of contexts in a significant manner. This is more pronounced in a modern textbook such as Maximum.

\subsection{Comparison Using the Technology Cognitive Demand Level-Local Organization 2}

The second task in consideration, in the context of praxeology, is offering learners good mathematical tasks. Therefore, zooming into the exercises in the textbooks, the cognitive demand levels are decided using the four categories of Smith and Stein [18]: MT = memorization $\tau_{8}$, PWOC $=$ procedures without connections $\tau_{9}, \mathrm{PWC}=$ procedures with connections $\tau_{10}$, and $\mathrm{DM}=$ doing mathematics $\tau_{11}$. That is to say, these are another type of techniques, unified by another discourse (technology $\theta_{2}$ ) called cognitive demand level and, hence, another local didactical organization (LO2) under ATD used for analyzing the data in this work. The percentile (i.e., the number of tasks demanding a particular cognitive level divided by the number of tasks in that chapter) is plotted against the textbooks in Figure 10.

As seen in Figure 10, another common characteristic of all the textbooks is that the tasks that require higher-level demands II (doing mathematics) are few compared to the number of tasks that require lower cognitive demand levels. More than $90 \%$ of the tasks in the $9^{\text {th }}$ grade mathematics textbook from Ethiopia are dominated by exercises that require lower-level cognitive demands, i.e., memorization $\tau_{8}$ and procedures without connections $\tau_{9}$. Among the Norwegian textbooks, the modern textbook Maximum (both $9^{\text {th }}$ and $10^{\text {th }}$ grade) significantly stand out in offering tasks with higher-level demands I (procedures with connections $\tau_{10}$ ).

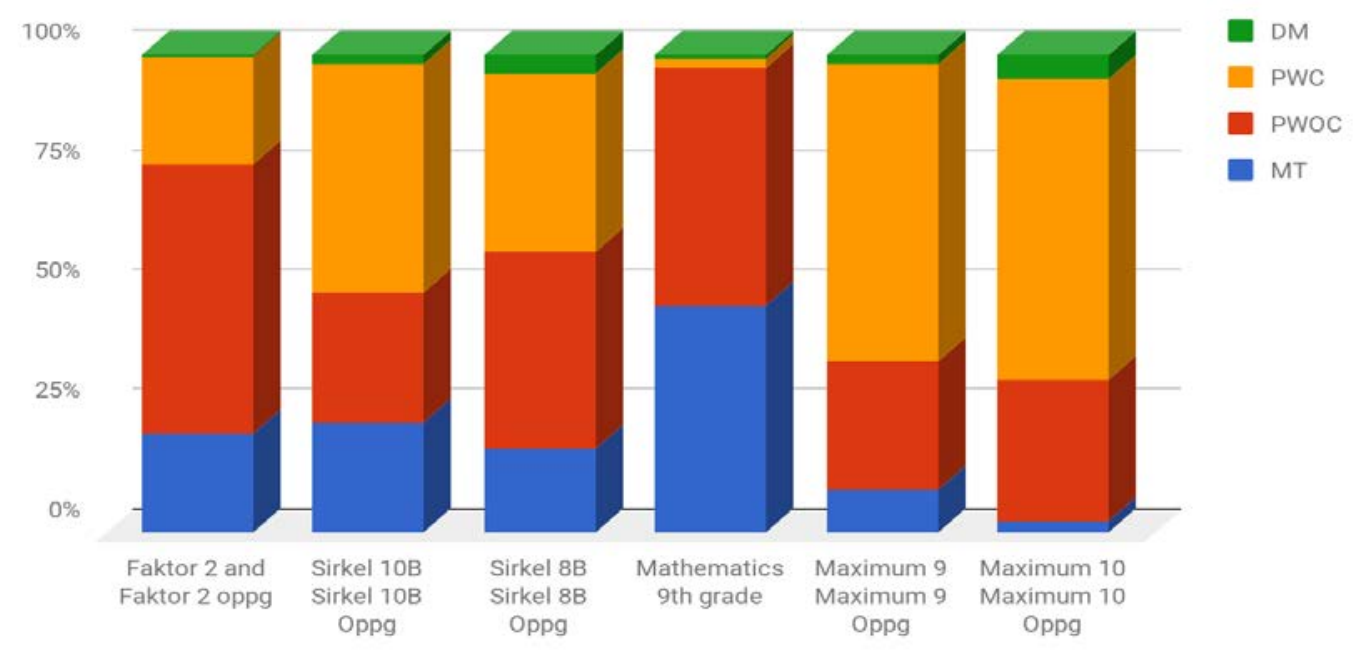

Figure 10. Percentile of the cognitive demand levels of the mathematical tasks (exercises) in different textbooks 


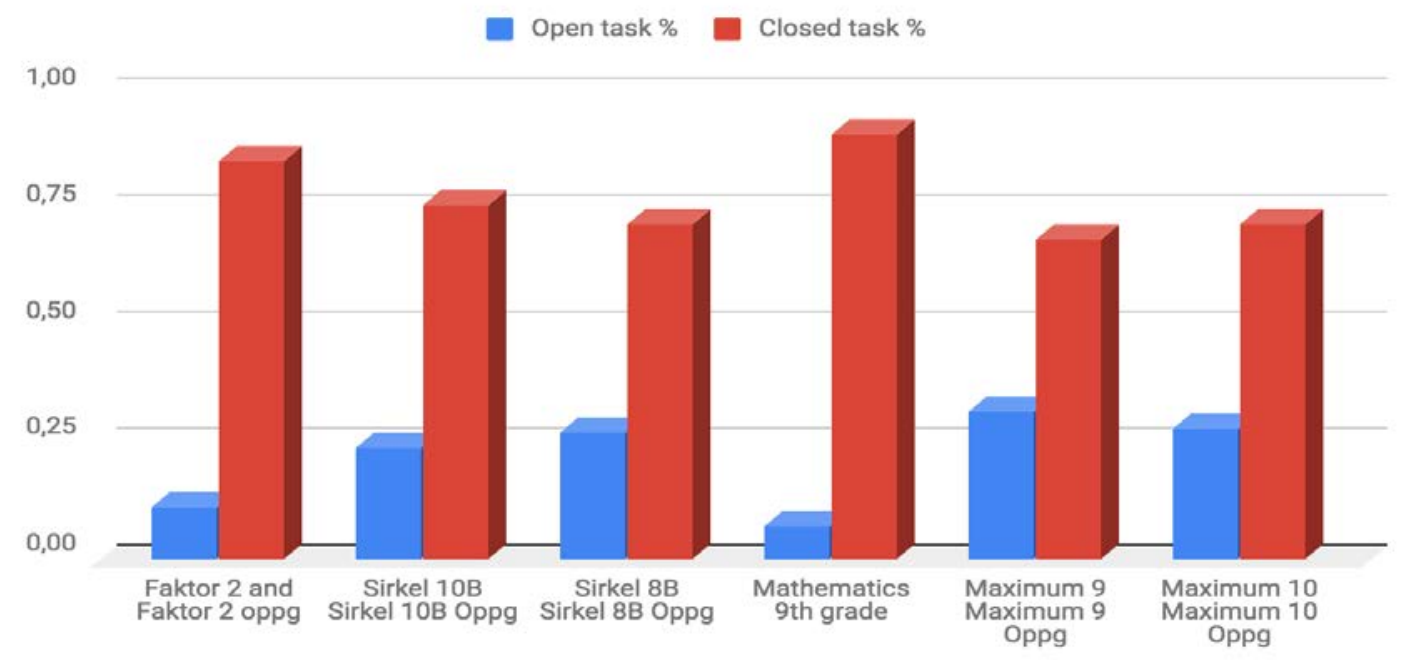

Figure 11. Percentile of the closed versus open-ended tasks of the different textbooks.

\subsection{Comparison Using the Technology Response Type- Local Organization 3}

The third didactic discourse (technology $\theta_{3}$ ) used for analysis is the discourse on mathematical tasks (exercises), that is, if the nature of their responses are open- and closed-ended. This means that the response type is the unifying technology $\theta_{3}$ for the third local organization (LO3). The techniques, closed-ended $\tau_{12}$ and open-ended $\tau_{13}$, used to create the exercises are counted using the characterization given in Figure 5 above and the result is summarized in Figure 11. The number of closed tasks overwhelm in all the textbooks in general. The Norwegian textbook Faktur and the $9^{\text {th }}$ grade mathematics textbook have an approximately similar proportion of open- and closed-ended tasks. The textbooks Maximum 9 and 10 as well as Sirkel 8B and 10B provide a higher proportion of open-ended tasks.

\subsection{Comparison Using the Approach of the Topics- Local Organization 4}

The textbooks differ in the approach by which they introduce relations and functions. The fourth task $T_{4}$ considered is about the approach of the topics. The 9th-grade mathematics Ethiopian textbook first deals with the concept of relations (definition, examples, domains, and range of relations and graphs of relations) and then proceeds to the concept of a function (definition, examples, domains, and range of relations and graphs of functions). Sirkel, the Norwegian textbook, deals with the concept relation in $8^{\text {th }}$ grade, a full chapter called "sammenhenger" in Norwegian, and later in $10^{\text {th }}$ grade comes the topic function. We consider this as one technique, $\tau_{14}$. On the other hand, Faktor and Maximum do not provide explicit chapters on the relation. It is presented together with function, and we count it as another technique, $\tau_{15}$. Moreover, the domain under which these topics are included under the discipline mathematics (see Figure 3) in the Ethiopian and Norwegian textbooks differ. For example, the definition of relation with one example is provided in Figure 7 from the Ethiopian textbook. The definition of relations is based on the prior knowledge of the Cartesian product of two sets. The prior knowledge for the teaching and learning of the concept relation is set theory.

That means, in the $9^{\text {th }}$ grade textbook, the concept relation is presented as an extension of the concept of set theory. Set theory is covered in the previous chapter of the textbook. Also, the concept of function is defined as a special type of relations- that no two ordered pairs have the same first-coordinates and different second-coordinates. In other ways, the prior knowledge for the teaching and learning of the topic of a function is the topic of relations. On the other hand, Norwegian textbooks do not deal with the set theory topic for the sake of introducing the concept of relations and functions. Instead, they build the teaching and learning of these concepts based on algebraic thinking - that is, from the relationships of different variables using real-life contexts. In Figure 8, a definition for proportionality, as part of relation/function is provided in Sirkel $\left(10^{\text {th }}\right.$ grade $)$. One example is provided using the context of scooter driving, and the relationship between distance and time is given. Actually, this is a difference in the use of the unifying theory $\Theta$ under the praxeology model in Figure 2. Relations and functions are unified under the domain set theory $\left(\Theta_{1}\right)$ as shown in the $9^{\text {th }}$ grade textbook from Ethiopia, and they are under algebra $\left(\Theta_{2}\right)$ in the Norwegian textbooks.

\section{Findings and Conclusions}

A comparative study is an investigation of similarities and differences of a construct under two contexts. The constructs under comparison are mathematics textbooks 
and the topic of relations and functions (main construct is textbooks; relations and functions are sub-constructs). The Norwegian and Ethiopian culture and education system are the two different contexts under consideration. Specifically, the textbooks that contain chapters on relations and functions are selected.

The ATD is used as a framework to situate, structure, and analyze this comparative study. As praxeology is a four-tuple $(\mathrm{T}, \tau, \theta, \Theta)$ in ATD, different tasks, techniques, technology, and theory were included in the study. By considering four tasks $T_{1}-T_{4}, 15$ ways of performing the tasks, called techniques $\tau_{1}-\tau_{15}$, were used to analyze the data. These were organized around four technologies $\theta_{1}$ $\theta_{4}$. In addition, two theories $\left(\Theta_{1}-\Theta_{2}\right)$ help us regarding the main organizing explanations. The praxeologies bound together by the technologies are also called local organizations; LO1 - LO4 are the main comparing tools. Since tasks deal with relations and functions, we also have MOs unified by these theories.

Turning to technology $\theta_{1}$, one of the significant observations in this analysis is how poorly the $9^{\text {th }}$ grade mathematics textbook is equipped when it comes to the technique $\tau_{1}$ called context in the chapter on relations and functions. It may not be fair to generalize this observation to other chapters of the textbook or to mathematics textbooks of other grades. However, it is possible to claim that relations and functions are mathematical concepts suitable to be represented with different real-life contexts. In fact, among the Norwegian textbooks considered in the study, those published recently make better use of technique $\tau_{1}$. This may be attributed to the impact of the recent research-based mathematics education in general.

Under technology $\theta_{2}$, there is some similarity between Faktur 2 and 9th-grade mathematics book in using technique $\tau_{9}$-with lower-level cognitive demand II-but $\tau_{11}$ or tasks with higher- level cognitive demand II are few in both textbooks. In fact, $\tau_{8}$ and $\tau_{9}$ dominate the tasks in most of the Norwegian textbooks. In addition, most textbooks are dominated by the use of $\tau_{12}$ - closed-ended-when it comes to the technology $\theta_{3}$-response type of tasks.

The analysis around technology $\theta_{4}$, the approach towards topics, which we call the local organization, LO4, deals with prior knowledge. It was done via two techniques: technique $\tau_{14}$ for presenting relations and functions separately as in the case of Sirkel 8B, 10B and $9^{\text {th }}$ grade mathematics book, and technique $\tau_{15}$ simultaneously dealing with the two concepts as in the case of Faktor and Maximum. Sirkel dedicates one whole chapter about relation ("sammenhenger" in Norwegian) in the $8^{\text {th }}$ grade textbook and another whole chapter on the topic of function in the $10^{\text {th }}$ grade textbook. Most of the textbooks in Norway, except Sirkel, avoid presenting the concept of relation separately before covering the topic of function. In contrast, the $9^{\text {th }}$ grade mathematics textbook from Ethiopia has a separate section on relations and functions under the same chapter. Dedicating a separate chapter or section for relations can help to lay down a deeper foundation for the teaching and learning of functions. Avoiding it may, in turn, create a narrow understanding of functions.

On the contrary, using set theory as prior knowledge for relations and functions seems outdated in Norwegian textbooks. Set theory as $\Theta_{1}$ is not used as a foundational concept in mathematics education in Nordic countries in general [16]. This may be due to the movement called the 'New Math,' where the set-theoretic way of approaching school mathematics have started to get less attention [39]. Now algebraic thinking is considered as the foundation for the concept of functions.

The local organizations LO1-LO4 above are determined mainly at level 6 of didactic co-determination in Figure 3. As Valverde et al. [7] deduced, textbook form and structure follow a distinct pedagogical model. This means that one of the reasons why the textbooks from Norway and Ethiopia is due to level 6 in Figure 3. Of course, we also observe difference at the domain level in the didactic co-determination; hence the different theories $\Theta_{1}$ and $\Theta_{2}$. In the Norwegian mathematics textbooks, relations and functions are extensions of the teaching of algebra or algebraic thinking $\Theta_{1}$. In contrast, in the Ethiopian textbook, set theory $\Theta_{2}$ is the domain that can encompass both relations and functions. At least, that is how the content structure is done in the $9^{\text {th }}$ grade mathematics textbook. Even further, the difference may come from level 7 (school), level 8 (society) and even level 9 (civilization). But this was not included in the study.

In general, many of the levels of didactic co-determination by [20] may have a direct or indirect impact on this comparative study on textbooks. For example, the difference at the civilization level could result in a difference in the design of the textbook since Ethiopia and Norway do not share a homogenous culture and are in different modes of civilization. But this is not pursued directly in this study. Similarly, state, society, or regions have an impact on designing the intended curriculum; however, this too is not investigated directly.

In Norway, many textbooks are provided by agreement of the authors of the text and the publishers. As far as one follows the guiding principles in the policy and the textbooks are sold, it is open for anyone. It seems that the authors and publishers adhere to the curriculum standards set by the policymakers. However, the way one develops a textbook is open to individual textbook author and publisher. The choice of a textbook to be used at a school is done at the school level in Figure 3 (by principals, teachers, and parents). While in Ethiopia, both the development of the textbook and the usage of the textbook are decided at level 8 (see Figure 3), by the government, and only one mathematics textbook is prepared for each grade.

According to the data from the direct textbook analysis using the didactical principles explained above, no direct observation of students learning or no assessment of 
mathematics knowledge of the students as in the study of PISA is not done. Hence, level 0 is not also included in this comparative study. The main focus of the study approximately lies between levels 3 and 6 . It also includes level 1 or 2 by focusing on definitions, examples, and exercises in the selected textbooks. It provides the comparison of textbooks from heterogeneous cultures and textbooks within the same culture at level 9. Finally, it is also safe to say that ATD is a powerful tool for comparative studies and can even be used as a research tool in designing textbooks.

\section{Acknowledgements}

We are very grateful to colleagues at the mathematics education section of Norwegian University of Science and Technology (NTNU) for their appropriate and constructive suggestions to improve this work.

\section{REFERENCES}

[1] J. Cai, I. Mok, V. Reddy, K. Stacey. ICME-13 Topical Surveys, 2016. 10.1007/978-3-319-42414-9.

[2] E. Jablonka, P. Andrews. CERME 7 Working Group 11: Comparative studies in mathematics education, Research In Mathematics Education, Vol. 14, No. 2, 203-204, 2012.

[3] M. Artigue, C. Winsløw. International comparative studies on mathematics education: a viewpoint from the anthropological theory of didactic, Récherches en Didactiques des Mathématiques, Vol. 30, No. 1, 47-82, 2010.

[4] M.B Bradburn, M.D. Gilford. A framework and principles for international comparative studies in education. Washington, DC: National Academic Press, 1990.

[5] D.J. Clarke. International Comparative Studies in Mathematics Education. In A.J: Bishop, M.A. Clements, C. Keitel, J. Kilpatrick, and F.K.S. Leung (Eds.), Second International Handbook of Mathematics Education, Dordrecht: Kluwer Academic Publishers, 145-186, 2003.

[6] S. A. Tesfamicael, D. Taye, G. Belay, A. Tulu, A. Tesfay. Comparative Study: The case of mathematics Teacher Preparation (MTP) programs in Norway and Ethiopia, 2018.

[7] G.A. Valverde, L.J. Bianchi, R.G. Wolfe, W.H. Schmidt, R.T. Houang. Physical Features of Textbooks. In: According to the Book. Springer, Dordrecht, 2002.

[8] S. Rezat. Learning mathematics with textbooks. In O. Figueras, J.L. Cortina, S. Alatorre, T. Rojano and A. Sepulveda (Eds.), Proceedings of the 32nd Conference of the International Group for the Psychology of Mathematics Education and PME-NA XXX, Vol. 4, 177-84. Morelia, Mexico: PME, 2008.

[9] J.T. Remillard. Examining key concepts in research on teachers' use of mathematics curricula, Review of
Educational Research, Vol. 75, No. 2, 158-75, 2005.

[10] B. Grevholm (2017). Mathematics textbooks, their content, use and influences. Research in Nordic and Baltic countries. Cappelen Damm Akademisk. ISBN 9788202566296. 514 s.

[11] L. Haggarty, B. Pepin. An investigation of mathematics textbooks and their use in English, French and German classrooms: Who gets an opportunity to learn what? British Educational Research Journal, Vol. 28, No. 4, 567-590, 2002. DOI: $10.1080 / 0141192022000005832$

[12] B. Pepin. CERME 6 Working Group 13: Mathematical tasks and learner dispositions: A Comparative perspective, 2504, 2010.

[13] S. Vinner, T. Dreyfus. Images and definitions for the concept of function, Journal for Research in Mathematics Education, Vol. 20, No. 4, 356-366, 1989.

[14] D.J. Denbel. Functions in the Secondary School Mathematics Curriculum, Journal of Education and Practice, volume and page numbers, 2015.

[15] H.K Nilsen. Learning and teaching functions and the transition from lower secondary to upper secondary school. Doctoral thesis, Kristiansand: University of Agder, 2013.

[16] O. Viirman. The function concept and university mathematics teaching (Doctoral dissertation). Department of mathematics and computer science, Karlstad University, 2014.

[17] Ö Hansson. Studying the views of preservice teachers on the concept of function. Department of mathematics, Luleå University of Technology, 2006.

[18] M. K. Stein, M.S. Smith. Mathematical tasks as a framework for reflection: from research to practice, Mathematics Teaching in the Middle School, Vol. 3, No. 4, 268-275, 1998.

[19] V. Floriano. Open-ended tasks in the promotion of classroom communication in mathematics, International Electronic Journal of Elementary Education, Vol. 4, No. 2, 287-300, 2012.

[20] Y. Chevallard. Organiser lTétude 3. Écologie \& régulation. In Dorier, J. L. et al. (Eds.), Actes de la $11 \mathrm{e}$ école de didactique des mathématiques. Grenoble: La Pensée Sauvage, 2002.

[21] C. Winsløw. Anthropological theory of didactic phenomena: Some examples and principles of its use in the study of mathematics education, 2011.

[22] L. Fan, Y. Zhu, Z. Miao . Textbook research in mathematics education: development status and directions, ZDM, Vol. 45, No. 5, 1-14, 2013.

[23] Z. Putra, G. Witri. Anthropological theory of the didactic: a new research perspective on didactic mathematics in Indonesia, 2017.

[24] Y. Chevallard. Fundamental concepts in didactics: Perspectives provided by an anthropological approach. In R. Douady and A. Mercier (Eds.), Research in Didactique of Mathematics, Selected Papers. La Pensée Sauvage, Grenoble, pp. 131-167, 1992.

[25] Y. Chevallard, M. Bosch, S. Kim. What is a theory 
according to the Anthropological Theory of the Didactic? In K. Krainer \& N. Vondrová (Eds.), Proceedings of CERME9 (this volume), 2015.

[26] M. Bosch, J Gascón. Introduction to the Anthropological Theory of the Didactic (ATD). In Bikner-Ahsbahs A., Prediger S. (Eds.), Networking of Theories as a Research Practice in Mathematics Education. Advances in Mathematics Education. Springer, Cham, 2014.

[27] D. Wijayanti, C. Winsløw. Mathematical practice in textbooks analysis: Praxeological reference models, the case of proportion, REDIMAT, Vol. 6, No. 3, 307-330, 2017. doi: 10.1783/redimat.2017.2078

[28] R. Lesh, T. Post, M. Behr. Representations and translations among representations in mathematics learning and problem solving. Problems of representation in the teaching and learning of mathematics, 21, 33-40, 1987.

[29] National Council of Teachers of Mathematics (NCTM). Principles to actions: Ensuring mathematical success for all. Reston, VA: Author, 2014.

[30] A. Sáenz-Ludlow, G. Kadunz. Semiotics as a Tool for Learning Mathematics: Springer, 2015.

[31] A. A. Siti. Comprehending the Concept of Functions, Procedia - Social and Behavioral Sciences, Vol. 8, 281-287, 2010. ISSN 1877-0428, http://dx.doi.org/10.1016/j.sbspro.2 010.12.038.

[32] R. Lesh, K.A. Cramer, H. Doerr, T. Post, J. Zawojewski. Model development sequences: Models and modeling perspectives on mathematics pr. In R. Lesh, \& H. Doerr (Eds.), Beyond Constructivism: Models and modeling perspectives on mathematics pr (pp. 35-58). Mahwah, NJ: Lawrence Erlbaum Associates, 2003.

[33] National Council of Teachers of Mathematics(NCTM). Principles and standards for school mathematics. Reston, VA: Author, 2000.

[34] L. Lesser, M. Tchoshanov. The effect of representation and representational sequence on students' understanding. In Lloyd, G. M., Wilson, M., Wilkins, J.L.M., \& Behm, S.L. (Eds.), Proceedings of the 27th annual meeting of the North American Chapter of the International Group for the Psychology of Mathematics Education, 2005.

[35] C. Janvier. Problems of representation in the teaching and learning of mathematics. Hillsdale, NJ, US: Lawrence Erlbaum Associates, Inc Problems of representation in the teaching and learning of mathematics. xi 247 pp, 1987.

[36] A.J.W. Van, K.S. Karp, J. M. Bay-Williams. Elementary and middle school mathematics: Teaching developmentally. Pearson Education limited, 2015.

[37] National Council of Teachers of Mathematics (NCTM). Professional standards for teaching mathematics. Re ston, VA: Author, 1991.

[38] J. Boaler. Open and closed mathematics: student experiences and understandings, Journal for Research on Mathematics Education, Vol. 29, No.1, 41-62, 1998.

[39] T Breiteig, S. Goodchild. (2010). The development of mathematics education as a research field in Norway. In. B. Sriraman, C. Bergsten, S. Goodchild, G. Palsdottir, B. Dahl, \& L. Haapasalo (Eds.), The first sourcebook on Nordic research in mathematics education: Norway, Sweden, Iceland, Denmark and contributions from Finland (pp. 1133). Charlotte, NC: Information Age Publishing, 2010. 